Siree oozing or any vessel be seen spouting, then the uterine vessels have not been properly secured, so a second ligature should be passed more deeply on one or both sides. 6. The next step (and one to which I attach great importance) is to cut out a V-shaped piece of the cervix and then to bring the flaps of the stump accurately and firmly together by means of a continuous deep silk suture, thus not only completely arresting any oozing from the stump, but also effectively shutting off any danger of sepsis from the vagina through the cervical canal, which is enclosed between the Haps. 7. Doubling in the peritoneal flaps (according to Lembert's method in suturing intestine) is important; it can be readily done, provided care be taken to cut the flaps, and especially the posterior one, long. Very often the stumps of the broad ligaments can be turned inside the peritoneal raps, so that at the completion of sewing up no raw surface of any kind whatever can be seen on looking into the abdominal cavity. 8. The most difficult cases are those in which the tumours burrow into one or both broad ligaments, for in these cases after the growths :are shelled out a more or less large cavity, or cavities, 'is, or are, left, in which blood is liable to collect, with danger to the patient. In Cases 15 and 18 the tumours burrowed between the layers of the broad ligaments, so that after I had removed the mass a cavity (into which an ordinary sized glass tumbler would have fitted) was left. This I treated in the following manner. Having tied all - oozing points, I whipped the sides of the cavity together by means of a continuous fine silk suture, commencing at the deepest part and proceeding upwards until the sac was obliterated effectually. Then the peritoneal flaps were brought together in the usual way. 9. In not a single instance was there the slightest necessity for the use of a drainage-tube. 10. It will be noted that in Cases Nos. 8 and 14 the tumours were comparatively small, and many operators would have been content with removal of the uterine appendages in order to stop the hæmorrhage and arrest growth; but I considered that having opened the abdomen I was not materially exposing the patient to more risk by performing hysterectomy as described; whereas, on the other hand, it is well known that in not a few cases removal of the uterine appendages has failed to bring about the desired results just mentioned. It was fortunate that I adopted the more radical "treatment in Case 8. This patient was sent in under my care by my colleague Mr. Henry Morris. The uterus being symmetrically enlarged and the hæmorrhage profuse, I thought she might have a fibroid polypus in the uterine cavity, so under an anæsthetic I dilated the cervical canal, and on exploring with my finger I found there was no polypus present, but a fibroid the size of a large orange embedded in the anterior uterine wall and pushing inwards the endometrium. A week later I performed intra-abdominal hysterectomy, and on laying open the tumour after its removal found in its centre a cyst the size of a Tangerine orange, so that had I been contented with removing the uterine appendages $I$ have little doubt that the fumour would have convinued to grow and thus have necessitated a second operation at some future date. 11. Suturing the abdominal walls in three layers is, I think, of sufficient importance to justify the extra time it takes. Since adopting it $I$ have not had a single case in which the scar has subsequently yielded, giving rise to $a$ ventral hernia. 12. Lastly, it seems to me that in the operation herein described we possess a means by which almost every class of cases of uterine fibroids can be effectively and safely dealt with; not only those which threaten life from excessive hamorrhage or dangerous pressure upon importint - organs such as the bladder or the bowels, but also those which give rise to discomfort by reason of their large size and compel those who are the subjects of them to lead more or less the lives of invalids.

Harley-street, W.

Railuay Mey's Convalescent Home at Sw. Lmonards.-On May 4th the Countess of Chichester opened the convalescent home which has just been erected at St. Jeonards in connexion with the Railway Mission. The building is of red brick with stone dressing, and is in an ezcellent situation overlooking the sea. The total outlay has been about $\$ 7000$, not including the value of the site. The site was purchased for $£ 600$ or $£ 700$ by Miss Dolby, who was in charge of the ola convalescent home at Hastings, and was presented by her to the committee.

\section{ACUTE PANCREATITIS.}

\section{BY R. M. SIMON, M.D. Cantab., F.R.C P.Lond.,} PHYSICIAN TO THE GENERAL HOSPITAL, BIRMINGHAM,

With a Pathological Note by

DOUGLAS STANLEY, M.D. Edin., M.R.C.P. LoND., IATE PATHOLOGIST TO THE HOSPITAL.

IT is possible that the subject of this paper is as great a novelty to many as it was to myself until I was brought into contact with a case in March, 1896. The most recent textbooks do not refer to it, and there have been from time to time references to isolated cases in British journals, but prior to 1889, when Dr. Fitz published the Middleton Goldsmith lecture in the New York Medical Record, there has been no attempt to collate cases or to systematise knowledge. It is to Dr. Fitz, therefore, that recent writers make reference, and to him that all credit for substantiating the existence of the disease should be given. It is possible, or even probable, that his classifications and conclusions need not be accepted in their entirety, but nevertheless his lecture will always remain as a classic and furnish the basis for further investigation. It would be well before discussing the pathology of the disease to give an account of the case which drew my attention to the subject.

CASR 1.-About midday on March 25th I was asked to see a man suffering from persistent vomiting of three days' duration. He had suddenly been seized with a most intense pain a little to the right of the middle line in the epigastrium. The pain quite disabled him and he had to give up his work, that of a night watchman, immediately, and take to his bed. He began to vomit and continued to do so almost without cessation until the time I saw him. I found him lying in bed with a bucketful of vomit by his side. He was a well-made but fat man of about forty years of age, and had, I believe, enjoyed very good health, but when I saw him he looked just like a man dying from unrelieved intestinal obstruction, or perforation of some abdominal viscus. His eyes were sunk and his face was pale and slightly jaundiced. His pulse was quick and feeble; the tongue was dry; and, above all, he had that terrible expression of anxiety that we are accustomed to associate with acute abdominal disease. The bowels had been confined from the first, and the urine was scanty. I turned down the bed-clothes with the confident expectation of finding the abdomen distended and tympanitic, but to my great astonishment there was no distension. The large intestine was obviously empty, and beyond a slight fulness in the epigastrium, which was not tender, there was no abnormality. It seemed a paradox that a man should present all the symptoms of intestinal obstruction and yet none of the signs. I could only conclude that the obstruction did exist but was very high up in the duodenum, or possibly the jejunum. There seemed only one possible explanation-viz., that a gail-stone had become impacted and was giving rise to the trouble. To this view some colour was given by the very sudden onset of the pain and by the slight jaundice. His friends were warned of the probable fatal result of all treatment, but were advised that the only hope lay in immediate operation. With this in view he was removed to the hospital, and Mr. W. F. Haslam, to whose kindness I am indebted for the following notes taken at the time of operation, opened the abdomen about four hours after admission. "The abdomen was opened by an incision in the middle line above the umbilicus, and coils of pale and very contracted small intestine were seen. On following the small intestine upwards this appearance was found to be present almost as far as the commencement of the jejunum. Here the intestine gradually increased in size and its colour changed from a pale slate colour to a redness that suggested inflammatory effusion into its walls, and the last piece of intestine that could be seen was of rather more than normal diameter, and its walls felt thickened. There were no signs of the intestine having been subjected to pressure by any constricting band, nor was anything found that could have pressed upon it. The lymphatio glands in the mesentery were greatly enlarged and in places calcareous, but they were not causing any pressure on the 
intestine. As the lumen of the intestine was found free from obstruction, and there was evidence of the duodenum being also free, the abdomen was closed. Operation gave no relief, and he died in a few hours."

Necropsy. - The body was found to be very fat, but bore no other morbid appearance. There was an operation in the mid-abdominal line. The thoracic organs showed no lesion of importance. There was peritonitis affecting the third part of the duodenum, there being much injection with deposit of lymph in a thin granular layer. There was some dark, opaque, greenish fluid in the cavity. The stomach was slightly distended. The mucous membrane was very catarrhal and showed ecchymoses in places and even acute gastritis in patches. Microscopic examination showed very marked catarrh of the mucous membrane, with considerable cellular infiltration of the sub-mucous and muscular coats. This infiltration was not evenly distributed, being more marked in some places, so that areas were seen under the microscope which contained great numbers of lencocytic cells and even fibrin. There was great engorgement of the ressels, with cell exudation in their neighbourhood. There was some dilatation of the duodenum and the wall was very much thickened. There was intense engorgement of the vessels and in flammation of the peritoneal surface, the mucous surface showing many ecchymoses. There was no obstruction of the duct, which was found to be quite pervious. In the cellular tissue (extra-peritoneal) there was some infiltration with opaque grey fluid. Microscopic examination showed intense inflammation of all the coats of the duodenum, consisting of fibrinous exudation into their tissues with a very marked amount of cellular infiltration as well. These cells were of various characters and sizes and very many of them showed karyokinesis. All the vessels were greatly engorged, and round them was a very marked cellular exudation. There was marked catarrh of the mucous membrane, and the cell infiltration mentioned above extended as far as the bases of the villi, and in some places seemed to have quite obliterated Brunner's glands; in others these glands could be seen lying in a mass of exudation and their epithelium appeared extremely catarrhal. A section was made showing the passage of the pancreatic duct through the wall of the duodenum, and its examination showed marked catarrh of the epithelial coat. The jejunum and ileum showed no lesion. There was nothing suggestive of intestinal obstruction. The iliac mesentery contained a cretaceous nodule of considerable size. All the mesentery seemed loaded to an abnormal extent with fat. The large intestine showed no lesion. The liver weighed $68 \mathrm{oz}$. The organ was enlarged, brown-red in colour, and the lobules were very distinct. There was no abscess formation or other lesion. The gall-bladder contained a single large cholesterine calculus and encysted cholesterine was found in the mucous membrane. Microscopic examination of the liver showed some fatty degeneration of the cells. The pancreas weighed $8 \frac{1}{2} \mathrm{oz}$. The organ was enlarged, soft, and of a peculiar greasy feeling at the head; towards the tail it seemed unaltered. The surrounding cellular tissue was infiltrated by an opaque grey fluid similar to that found outside the duodenum. This infiltration seemed to penetrate the pancreas, passing into the interstitial tissue, but forming a soft, almost putty-like exudation, which gave to the head the peculiar greasy feeling and swollen appearance already mentioned. Almost all the lobules of the head portion were surrounded by this substance. The glandular portion also appeared to be swollen and slightly translucent. Microscopic examination of the head of the pancreas showed that all the interlobular septa were infiltrated by fibrinous exudation in which were cells in greater or less proportion showing proliferative or destructive changes. In some places these cells were extremely granular and stained badly, in others their nuclei were more distinct. In more affected portions these cells appeared to have invaded the gland acini, and the glandular epithelium showed necrotic characters. Beyond this it seemed merely swollen. In the less severely affected parts there was no infiltration of the gland tissue by the cells, the exudation being confined to the interstitial portions of the pancreas. In many places this connective tissue showed, not infiltration by exudation, but marked proliferation, and the walls of the ducts and even those of the vessels were much thickened. The duct epithelium showed distinct catarrhal change in these situations. On passing towards the middle of the pancreas these changes became much less marked and examination of the tail end failed to show any morbid condition, there being no exudation into the connective tissue or even proliferation of its cells. The gland tissue also appeared to be quite healtbr. The right kidney weighed $6 \frac{1}{2} \mathrm{oz}$. and the left $6 \frac{3}{4} \mathrm{oz}$. The organs were very mottled and the vessels very engorged. Tho cortex was swollen. The capsule stripped fairly well. The pelves were healthy. Microscopic examination showed some catarrh of the tubules. The suprarenals were small, flabby, and very p'gmented. On microscopic examination they sbowed fatty degeneration. The spleen weighed $9 \mathrm{oz}$, was soft and pultaceous. Microscopic examination showed no special characters.

CASE 2.-The second case, which I have the kind permission of Dr. E. Rickards to make use of, was almost iden tical in its history and similar in its treatment and result.

Necropsy. - The body was also found to be very fat. The peritoneum showed some inflammation over the anterior sur. face of the liver and round the pylorus, being slightly opaque and dull. This seemed to be due to extensive infiltration of the retro-peritoneal tissue by opaque brown-grey fluid, there being at the same time considerable cellulitis with deposit of greasy, pultaceous material similar to that found in the first case. There were areas of "fat necrosis" on the great omentum and otber parts of the peritoneal surface in the neighbourhood of the pancreas. The pancreas was much enlarged $\left(8 \frac{1}{2} \mathrm{Oz}.\right)$, and showed appearances very similar to those found in the first case, only in a more marked degree the same pultaceous infiltration being found. There were; however, areas showing necrosis in the most affected portions of the head, together with small hæmorrhages (see illustration). As in the first case, the head of the pancreas was the

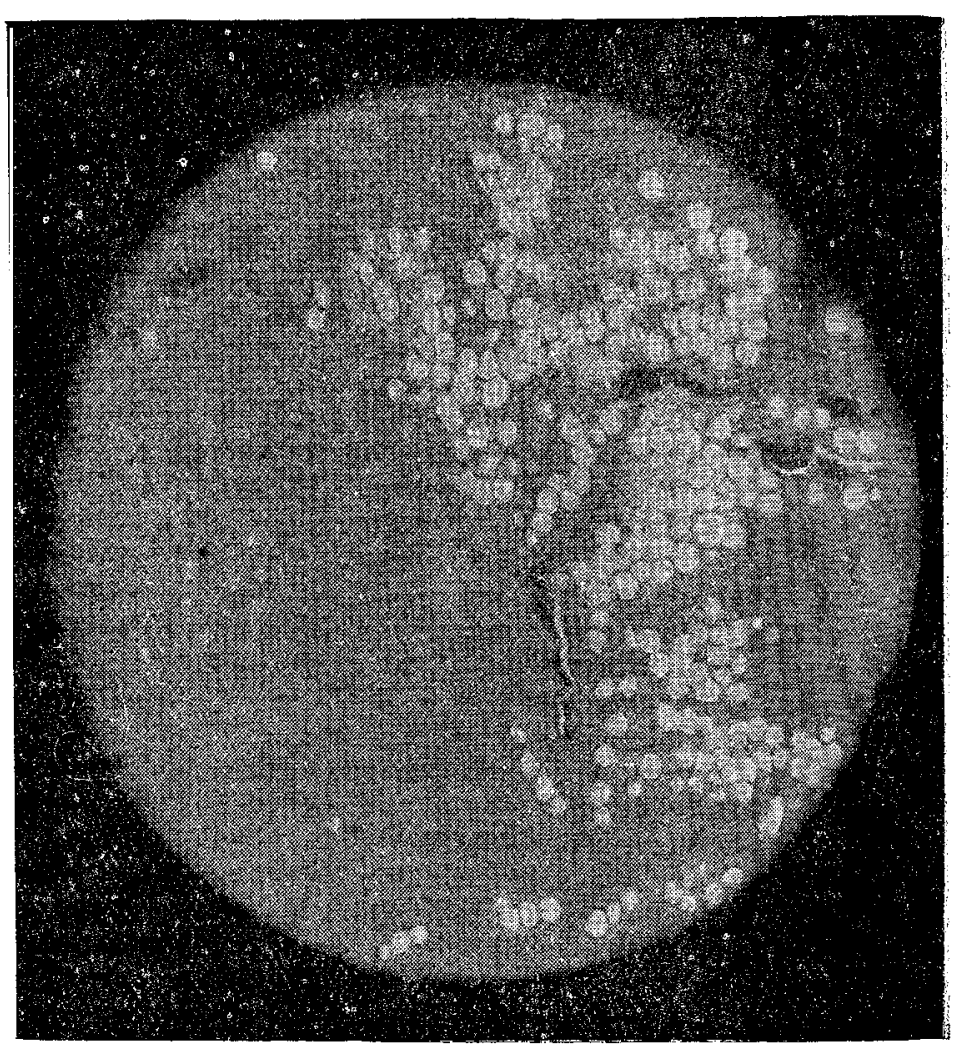

Section of pancreas near head, showing inflammatory infiltration hæmorrhages, ;and small patches of necrosis.

portion showing the most intense changes, the tail being comparatively unaffected. The microscopic appearances were also the same except for the presence of necrosis and bæmorrhages. The duct also showed considerable catarrhal change of its epithelium. The stomach showed much more severe changes than in the first case, being more bæmorrhagic, and there even seemed to be actual necrosis of the mucosa. The microscope confirmed these changes. The duodenum also was acutely inflamed and showed much swelling of its coats, and the same intense infiltration as described in the first case was found. The spleen, liver, suprarenals, and semilunar ganglia showed no important changes, the latter even appearing quite bealthy.

CASE 3.- The third case was that of a stout woman, aged sixty-three years, under Mr. Heaton's care in the General Hospital, Birmingham. When admitted she complained of pain and distension of the abdomen, with constipation and vomiting. The duration was five days. Five days ago the patient had pain in the right loin and iliac fossa, spreading across the abdomen. Her bowels were opened on that day. Later in the day she began to romit. Since then she had 
Fad constipation with increasing distension of the abdomen and pain. The pain, however, had been rather less than at the onset. She had passed urine normally. Notwithstanding the stoutness of the patient, the tissues were firm and not flabby to the feel. The skin was slightly yellow. The face was drawn and rather anxious in expression. She looked ill. The tongue was large, moist, and furred in the middle. The temperature was $99^{\circ} \mathrm{F}$. The pulse was fairly good. The abdomen was large, distended and soft; there was no tenderness. On percussion, it was tympanitic asteriorly, duller in the flanks, more expecially on the right side. There was no fluid. There was discolouration of skin cound the umbilicus, but no signs of hernia, or anything abnormal on palpation. The patient had vomited more or less continuously since the first onset. The vomit, said to have been ordinary at first, was now a fæcal and dark brown fluid. The patient stated that she had had a somewhat similar attack two years previously, but not so severe. She had not suffered from chronic constipation. On examination per rectum nothing abnormal could be felt. The patient was put under an anæsthetic. An incision was made in the middle line, the peritoneum opened, and the bowels exposed. The intestines were not much distended, and appeared rather redder than enormal. There was no sign of inflammation in the right iliac iossa. No strangulation or obstruction was found. The patient got weaker and weaker during the operation, with fxcal vomiting, and died soon after its completion.

Necropsy. - The body was extremely fat. On the peritoneal surface were scattered patches of a grey colour, abjut $\frac{1}{8}$ in. in diameter, and evidently areas of fat necrosis. There was some fibrous thickening round the pancreas as from some former inflammation. The pancreas was enormously enlarged and contained large extravasations of blood, there being patches of well-marked necrosis outside the areas of nemorrhage as well. This condition involved the whole organ, and outside the pancreas there was also hæmorrhage, this appearing to extend down in the retro-peritoneal tissue almost into the pelvis. There was a cystic cavity at the apper margin of the pancreas containing about an ounce of bloody, brownish fluid. The daodenum, though compressed, was not inflamed. The other organs showed no special change.

In the three cases bacteriological examination of the tissues of the pancreas and the fluid contained in the surrounding cellular tissue showed the presence of microorganisms of different character. One of the most distinctive was a bacillus somewhat slender, with rounded ends, and which does not stain by Gram's method. Bacterium coli commune was also obtained by culture, especially from the fluid surrounding the pancreas and extending down behind the colon. These cases are, therefore, almost identical in their morbid anatomy, the only difference being of degree. Taking into consideration the manner in which the stomach and duodenum showed intense inflammation with involvement of the head of the pancreas-i.e., the adjacent portion-the tail practically escaping, the question may be raised as to whether we may not have to deal with an infective gastro-duodenitis, extending into the surrounding tissues. The manner in which the retro-peritoneal conmestive tissue showed infiltration and inflammation obviously from extension downwards by gravitation from the seat of the most severe lesion round the duodenum at once suggests som 9 infective condition, and the abundance of micro-organisms in all the affected parts is of great importance. That the pancreas is not necessarily the primary organ affected is very probable, and from its situation and texture it would be readily affected by any infecting organism, either from the pancreatic tissue or by way of the duct. As this latter opens directly into the duodenum it is plain that any infecting organism could find a way into the organ. On the other hand, in many cases the pancreas seems to be the only organ affected, as in Case 3.

Here, then, we have a group of three cases which during Mife were thought to be characteristic of intestinal obstruction, or at least so nearly characteristic that, though the condition of the patients was clearly desperate, it was felt not to be right to withhold from them the chance that laparotomy might give. In my own case there was no general distension; this was present in Mr. Heaton's case, and the abdomen was tympanitic; but as a rule in recorded cases no general distension is present, and it may be possible that this distinction will help us in arriving at a diagnosis in the difficulty such a case presents. Fitz divided his cases into the following groups: (1) pancreatic hæmorrhage ; (2) bxmorrhagic pancreatitis; and (3) acute pancreatitis which might stop short of or reach a gangrenous condition. Pathologically our cases correspond in inverse order to these divisions, but clinically no such division is possible, as it is only when the shock becomes very great or symptoms of intestinal obstruction occur that the case, as a rule, comes under medical observation. There are, I believe, no pathognomonic symptoms which can lead one in the present state of knowledge to diagnose or even suspect the presence of pancreatitis before the changes become pronounced and the condition hopeless.

In the case of very great hæmorrhage (Case 3) there were evidences of old adhesions at the back of the pancreas, and therefore our ignorance of the earlier stages of the disease may be only temporary, and we may argue from a fact like this that there had been an abortive or slight attack of the disease fire years before the fatal attack. It would be the greatest unwisdom to disturb ourselves and alarm our patients by the suggestion that pain in the epigastrium and vomiting might mean a rapidly fatal disease, but the persistence of these symptoms without adequate cause should certainly in some cases excite very great apprehension. When we ask ourselves what all the fatal cases of pancreatitis have in common besides the pancreatic lesion, we find that it is neither sex, occupation, nor previous history of illness. It is not even, as we might expect, recent gastro-duodenitis, for there are several cases of large pancreatic hæmorrhage recorded in which death occurred suddenly and without warning by symptoms. In every case, however, the patient has been fat, and in a large proportion of the cases has become rapidly fat, and showed on post-mortem examination a heavy, fat omentum. In addition, every case in which proper examination has been made has shown the presence of numbers of differing kinds of bacteria. It is probable that in these two conditions we have both a predisposing and an efficient cause for the disease. Anything which delays the products of digestion in the duodenum, especially if these be decomposing, will increase the liability of infection of the pancreas, and according to the virulence of the infection will be the acuteness of the disease and inversely the duration of life. One cannot look at the duodenum without thinking that its shape, while necessary for delay in the passage of food in order to get due admixture of bile and pancreatic secretion, may become a source of danger if any addition to the delay in the passage of the products of digestion or decomposition arises, as must result from the presence of a large amount of omental and mesenteric fat, and therefore it is that epigastric pain in fat people, or those who are somewhat rapidly becoming fat, assumes an importance both from the view of treatment and from that of prognosis. No one can see a case of acute pancreatitis without being oppressed by the horror of its suddenness, of its severity, and its hopelessness. A sudden but sometimes fearful pain in the upper part of the abdomen, shock which may be so great as to kill the patient with the rapidity of cerebral hæmorrhage, and vomiting which continues unrelieved until stopped by deaththese constitute a trio of symptoms before which we are hopeless. But is there nothing we can do to prevent their occurrence? I fear not if, as seems most likely, the disease is due to infection by a pathogenic organism. It is possible that by treating vigorously gastro-duodenal catarrh, and by means of salol and other disinfectants rendering the duodenum an unsuitable habitat for bacilli, we may diminish the likelihood of pancreatic infection, but until the symptoms of pancreatitis occur we can only guess at its existence, and its nonoccurrence cannot furnish an argument that our treatment has averted it. When once the disease has commenced, no treatment has been effectual. In common with nearly all recorded cases, those in the General Hospital, Birmingham, were treated by laparotomy, and to my thinking properly so. There must be an element of doubt, even if pancreatitis be suspected, and the doubt that must exist in every case as to whether or not we have to deal with intestinal obstruction from the impaction of a gall-stone or from other causes will justify operative interference when relief by drugs has been despaired of.

Birmingham.

The Use of CaLF Lymph.-The Horsham board of guardians has decided to supply its medical officers with calf lymph for the public vaccination of children subject to the consent of the Local Government Board. Most of the medical officers of the board wrote in favour of such a plan. 